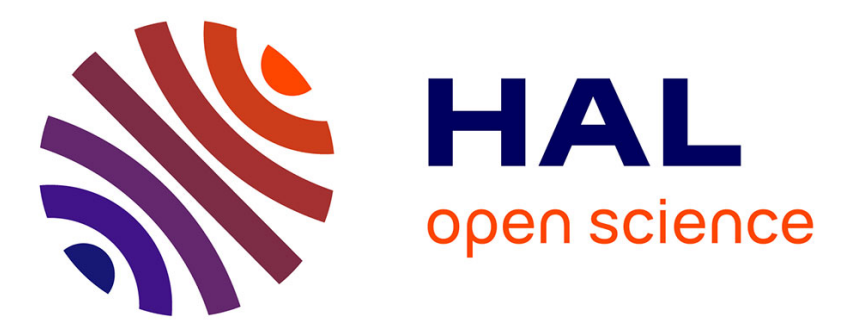

\title{
Generator of Road Marking Textures and associated Ground Truth Applied to the evaluation of road marking detection
}

\author{
Marc Revilloud, Dominique Gruyer, Evangeline Pollard
}

\section{To cite this version:}

Marc Revilloud, Dominique Gruyer, Evangeline Pollard. Generator of Road Marking Textures and associated Ground Truth Applied to the evaluation of road marking detection. IThe 15th IEEE Intelligent Transportation Systems Conference, IEEE, Sep 2012, Anchorage, United States. hal00735321

\author{
HAL Id: hal-00735321 \\ https://hal.inria.fr/hal-00735321
}

Submitted on 25 Sep 2012

HAL is a multi-disciplinary open access archive for the deposit and dissemination of scientific research documents, whether they are published or not. The documents may come from teaching and research institutions in France or abroad, or from public or private research centers.
L'archive ouverte pluridisciplinaire $\mathbf{H A L}$, est destinée au dépôt et à la diffusion de documents scientifiques de niveau recherche, publiés ou non, émanant des établissements d'enseignement et de recherche français ou étrangers, des laboratoires publics ou privés. 


\title{
Generator of Road Marking Textures and associated Ground Truth
} Applied to the evaluation of road marking detection

\author{
Revilloud Marc, Gruyer Dominique, Pollard Evangeline
}

\begin{abstract}
To increase driving safety, many researcher works on Advanced Driving Assistance Systems (ADAS) have been developed and embedded in real prototypes during the last decades. For some of these applications like Lane Keeping System, lane perception is an essential task. For others applications like Emergency Brake Assist, lane perception modules provide useful information helping the system to select only the most dangerous obstacles. Proposed solutions to perform lane detection become more and more elaborated, however no generic solution has been proposed to calculate performances of these algorithms. Lots of solutions have been proposed to perform this lane detection. However, no generic solution has actually been proposed to quantify the quality of such applications. It is appearing that this evaluation task is now very important and critical. Most of the existing evaluation stages can be classified in two main parts. In the first case, evaluation is based on natural images databases with ground truth of road marking and/or geometrical truth of lanes. In the second case, evaluation uses virtual data and simulated images. The first one is relatively hard to perform because it is based on manual labeling of natural images. The second one has automatic labeling clustering but a realistic virtual environment is required and more precisely both realistic road bitumen and road marking textures. This paper presents an efficient solution in order to simulate roads environment for the evaluation stage of road marking detection algorithms. Moreover, a powerful tool dedicated to the road marking texture generation is proposed. It takes into account both imperfection and wear of the road marking. A virtual database using this tool will be applied on a set of road marking extractor to validate the evaluation process with our virtual approach.
\end{abstract}

\section{INTRODUCTION}

$\mathrm{I}_{\mathrm{n}}^{\mathrm{n}}$ ntelligent vehicles are a major application to make transport safer and to improve the comfort of human. Simulation is a practical solution to prototype assistance systems or autonomous vehicles. Different situations including dangerous ones can be quickly tested in complex scenarios.

However, in most cases, the shift from simulation to testbed causes differences in outcome. This is due to a

Marc Revilloud and Dominique Gruyer are with the Laboratory on the Interactions between Vehicle-Infrastructure-Drivers laboratory (IM-LIVIC), of IFSTTAR Institute. 14 route de la Minière, bat 824, 78000 Versailles, France. (name.surname@ifsttar.fr).

Evangeline Pollard is with the INRIA institute (IMARA team). Domaine de Voluceau - Rocquencourt, B.P. 105 - 78153 Le Chesnay, France. evangeline.pollard@inria.fr. striking lack of realism of the surrounding environment and of data coming from virtual embedded sensors.

This study takes part in the work in progress about the improvement of the realism in simulation, and especially, for applications such as ADAS.

ADAS applications are generally split into three tasks: (1) the perception task, where data coming from exteroceptive and/or proprioceptive sensors are processed in order to assess the environment, (2) the decision, where the output of the perception task is interpreted in order to define an adapted action, (3) the control that commands the actions on the vehicle.

Our study focuses on the generation of data in order to obtain an accurate environment simulation. This realistic virtual environment should be used in order to prototype algorithm for the perception task. More precisely, the perception task addressed in this study is the lane and road marking detection.

In this context, the goal is to provide information about the geometry of the road (position of lane, curvature, etc.). Most of these road marking detection algorithms are split in three steps. In the first one, images are processed to extract road marking primitives. In the second one, geometry of the traffic lane is estimated by studying the distribution of extracted primitives. Finally, temporal monitoring of parameters allows tracking and filtering the estimations in a video sequence [1].

Protocols have been proposed to evaluate parts of lane marking perception. [2] proposed an evaluation of the road marking extraction, base on a reference database containing natural images. However, validation based on reference database images cannot test the robustness of these extractors independently of the road configuration. In previous work [3], an evaluation based on synthetic images from the SiVIC platform (sensor simulation) was proposed. However in this previous work, only one marking texture was used to generate road marking. It leads to redundancy problems and it took not into account the possible wear of road markings.

The objective of this paper is to present a solution to generate realistic road marking textures based on natural bitumen texture, in order to build simulated video sequences. This process takes into account the road marking shape and a set of different types of wear identified by prior research 
on road markings wear. The result of this process is both a road marking texture and their associate ground reference.

The paper is organized as follows. First, in section II, the SiVIC platform and realism level of its simulation will be described. Then, Sect. III introduces road marking feature extraction algorithms and evaluation. In Sect. IV the traffic lane generator will be presented. In Sect. V, robustness of road markings feature extractions are compared and discussed. Finally, Sect. VI provides conclusions and perspectives.

\section{THE SiVIC PlATFORM}

SiVIC is a virtual sensor simulation platform (cameras, odometers, telemeters, GPS, etc.) involving a complex vehicle modeling. This simulation platform is dedicated to the prototyping, the test and the validation of ADAS solutions [4]. It was developed to test system boundaries. It reproduces a situation as realistic as possible (Fig. 1), as well as the behavior of a vehicle and its embedded sensors. In this environment, this vehicle is defined as an ego-vehicle. The simulation includes a road environment with a road, lanes, objects (trees, building, other interacting vehicles...) and an ego-vehicle. As SiVIC also integrates physical car modeling, the movement of the embedded camera can be realistically reproduced.

Among all the scenarios available in SiVIC, the most suitable for lane detection is probably the Satory's test track in Versailles. Indeed, this track is generated according to a virtual map of the Satory's Tracks. The geometric ground truth is provided by an accurate map (build from a centimetric GPS-RTK).

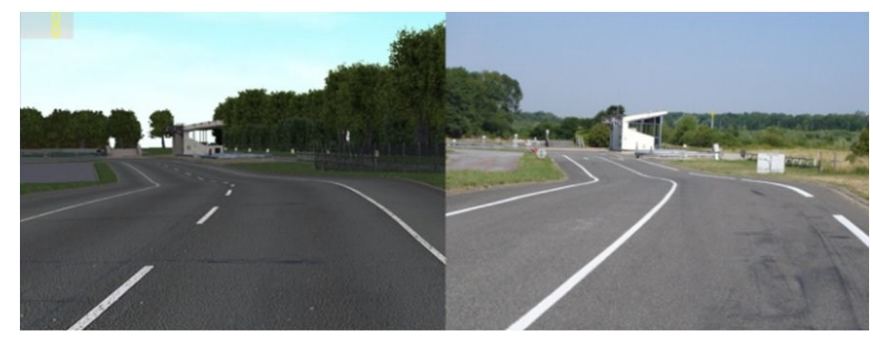

(a)

(b)

Fig. 1: (a) Simulated images (b) Natural images of Satory's Tracks

Another advantage of SiVIC is its capability to provide both simulated camera images and their associated ground truth. In Fig 2, the camera sensor images are simulated on the upper left image and their corresponding ground truth are simulated on the upper right image. Each marking line is labeled as a left, middle or right line.

In order to perform a realistic camera simulation, we deal with both an accurate modeling of the real optical sensor and with a realistic 3D model of the scene. The first point has already been studied and validated in [5]. Concerning the second one, we consider that the GPS-RTK, used to provide the road geometry ground truth of the Satory's test track, has a centimetric accuracy.

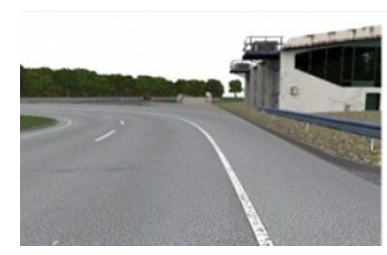

(a)

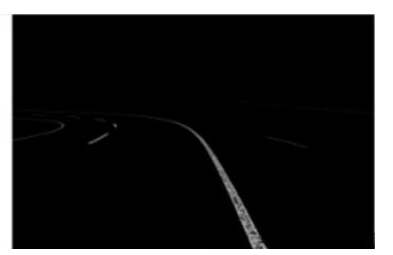

(b)
Fig 2: (a) Satory's test track (b) ground truth

Moreover, the various buildings and road sign elements have been accurately modeled and located in order to provide accurate data for the prototyping of perception algorithm (obstacles detection and tracking). Finally, road textures are based on the use of natural images coming from real road surface.

However, the problem of realism of these simulations is double. The first bad effect is due to the repetition of road marking textures during a camera capture. The second limit is due to the limited number of these textures proposed by sensor simulation dedicated to transportation issues. The aim of this paper is to propose a solution in order to limit these deficiencies and to test different levels of road marking quality with the same scenario.

\section{EXTRACTION ALGORITHMS AND VALIDATION}

Several techniques might be used to extract road marking features. Some of them are based on LIght Detection And Ranging (LIDAR) analysis due to the reflection energy (white lane markings reflect more energy than the road) [6]. But the most common way to extract road marking feature is probably camera [6].

Most efficient algorithms are based on variations in local grey level. They process each line of the images independently and detect black-white-black transition. The width of the transition for a line is computed as the projection of the road marking width from a top view to the frame view. An example of road marking feature extraction is shown in Fig. 3.

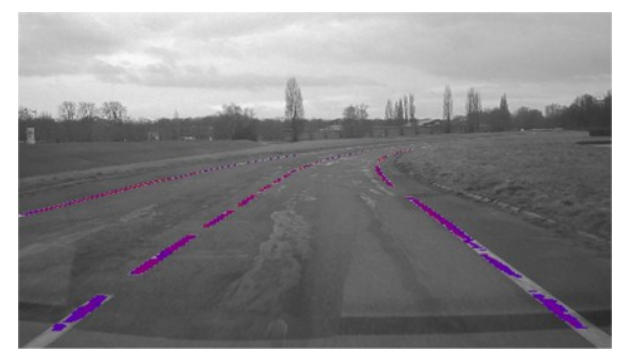

Fig. 3: Road marking feature extraction

To compare performances of these algorithms, [2] proposed an evaluation metric for all extractors rely on a detection threshold $T_{g}$. This evaluation tests the performance of the algorithms for all possible $T_{g}$ values in the range [0, 255]. Results are given by a Receiver Operating Characteristic (ROC) curve and Dice Similarity Coefficient (DSC). ROC curve corresponds to the ratio between True Positive Rate and the False Positive Rate. DSC curves allow solving the ambiguity in case of crossing curves. 
However, this evaluation metric is generally used on a small set of natural images. Very often images belonging to these image bases are timely and spatially independent, with different image quality and format. Moreover, it is very difficult, in real conditions, to generate a similar road scene with different road textures. With these limitations, it is almost impossible to efficiently evaluate the robustness of an algorithm independently of the road configuration and over time.

\section{TRAFFIC LANE GENERATION}

\section{A. Overview}

The proposed approach is based on road marking texture generation according to the influence of natural bitumen textures.

Road marking is characterized by both structural information (continue or discontinue shape, width of the marking) and textural information (different wear and techniques for painting application).

The whole process is divided into two stages. The first one consists of the generation of a binary mask that corresponds to the desired shape. The mask is used in order to set the positioning of road marking textures. The second stage is more challenging, because the goal is to generate a realistic road marking with different states of wears. These wears are precisely identified and presented in [8].

\section{B. Generation of road marking shape}

The road surface marking used for traffic lanes information are usually characterized by many attributes depending on country standards. In the current study presented in this paper, only French specifications will be addressed. These following attributes will be taken into account: the type of road, the color of road marking, the modulation type (T1, T2, etc.) and the road marking width. French specification attributes are shown in Fig 4. They are used to generate a binary mask that could be considered as "generic shape" ground truth in case of shape-based validation approaches.

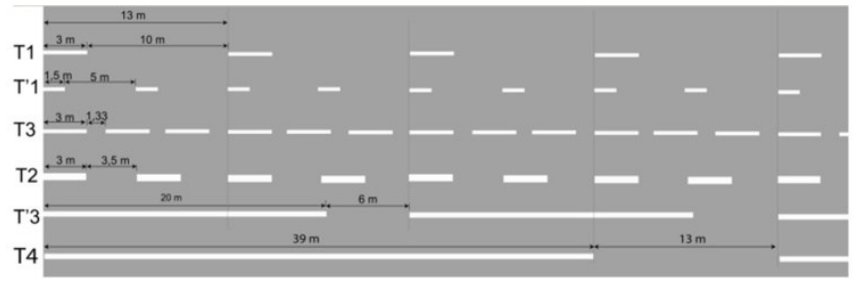

Fig 4. French road traffic lane description and specification

Indeed, in other cases, it is more appropriate to manage a more accurate ground truth based on "pixel-based" validation. This second approach does not take into account the wear that is responsible of mark disappearance (holes in the road markings).

\section{Pixel-based ground truth generation}

Two types of degradations cause disappearance of road surface marking: the tear out (Fig. 5a) and the cracking (Fig. $5 b)$.

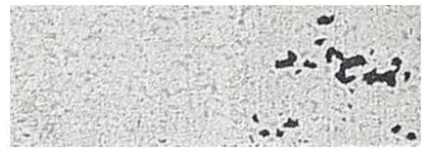

(a)

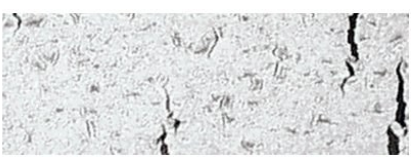

(b)
Fig. 5: (a) Tear out, (b) Cracking

Since painting composition of road marking was modified in the last decades (regulated by the Decree of 10 May 2000 on conformity of products), the phenomenon of cracking only due to the paintings tends to disappear. As a consequence, we focus on the tear out degradation. We choose to model the tear out using a pseudo-random noise. In practice, the spatial distribution of the holes on the road marking cannot be modeled by a classical statistical distribution. In order to avoid noise discontinuities and to obtain a realistic spatial distribution, Perlin noise seems to be the most adapted noise and will be used [9].

Perlin noise is usually used in computer graphics in order to build a multidimensional noise. It is based on a sum of several signals (oct: number of signals) at different frequencies (freq) weighted according to a persistence criterion (pers). It is particularly well suited to our problem, because it takes into account different frequency information (large or small holes) and persistence parameters (regularity of holes). It allows a better matching distribution of holes in road markings. By using this $2 \mathrm{D}$ Perlin noise in the interval $[-1,1]$ with an additional threshold $\left(t_{h}\right)$, the texture as shown in Fig. 6 is obtained. This threshold keep the pixel value if lower than a fixed value $t_{h}$.

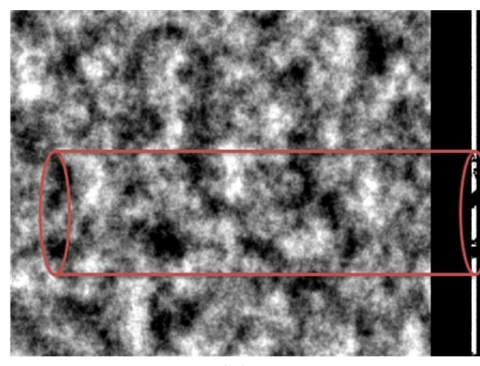

(a)

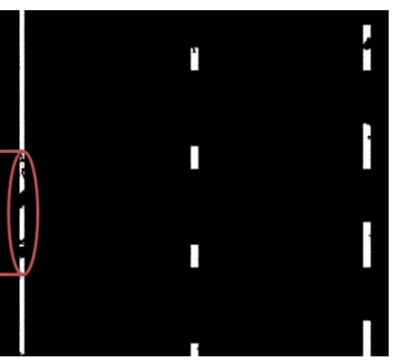

(b)
Fig. 6: (a) Perlin noise (oct $=6$, pers $=0.66$, freq $=1)$. (b) Generated gound truth associate $\left(\boldsymbol{t}_{\boldsymbol{h}}=-0.7\right)$

As shown in Fig. 6, when the Perlin noise is below the threshold $t_{h}$, the road marking pixel is removed.

In order to avoid smooth edges, a non-linear contour filter is performed. It is based on a random permutation of a contour pixel by one of its neighbors. This filter is set according to two parameters: the proportion of modified pixels $\left(\mathcal{P}_{m}\right)$ and the pixel neighborhood size $\left(\mathcal{N}_{l}\right)$.

An example is given in Fig. 7.

The resulting texture can be used as a "pixel-based" ground truth to validate algorithms for road marking feature extraction, such as MLT or SLT extractor that were cited above. Note that in [2] a similar approach has been used. 


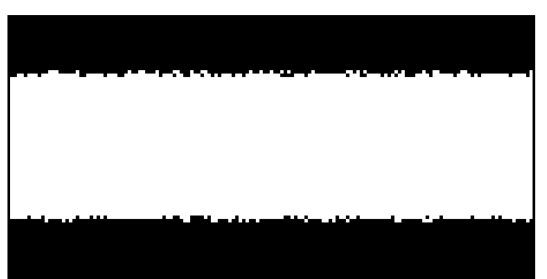

Fig. 7: Neighbor random noise $\left(\mathcal{P}_{\boldsymbol{m}}: 75, \mathcal{N}_{\boldsymbol{l}}: 1\right)$

\section{Texture degradation}

The aim of this task is to generate a visually realistic texture using both natural textures of bitumen and the mask texture (binary shape of the road marking) described in the previous section $\mathrm{C}$.

Texture degradations on road marking can be classified into three parts: the impact of bitumen, the dirt and the uniform wear. Each part is described in the next subsections.

1) Impact of bitumen on road marking

Road surface marking often depends on the nature of the bitumen as shown in Fig. 8.

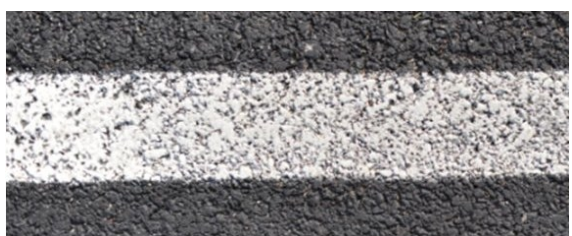

Fig. 8: Texture of bitumen with road marking

To take into account this dependence, we deal with a model based on the difference between local standard deviation and bitumen pixel value. The main advantage of this model is to maintain color variation of road marking similar to that of bitumen texture.

Let $p(x, y)$ be the resulting texture pixel value as:

$$
p(x, y)=c-\mathcal{P}_{b} \cdot\left(\overline{z_{l}}+\sigma_{l}-t(x, y)\right)
$$

Where $(x, y)$ are pixel coordinates, $c$ is the color of the road marking, $\mathcal{P}_{b}$ the impact factor of bitumen in interval $[0,1], \overline{z_{l}}$ the local pixel mean, $\sigma_{1}$ the local pixel standard deviation and $t(x, y)$ a pixel of bitumen natural texture in interval $\left[-\sigma_{1}, \sigma_{1}\right]$. The local pixel mean $\bar{z}_{l}$ is defined by a square neighborhood with a width equal to the width of the road marking.

\section{2) Dirt on road marking}

Sometimes, road markings are soiled. The dirt can be provided either by structured sources (tire marks) or not (dirt from the ground) as shown in Fig. 9.

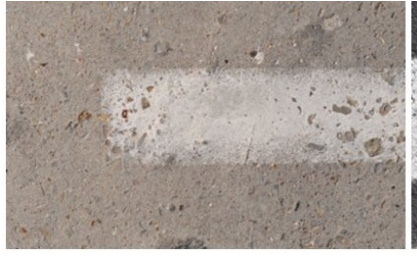

(a)

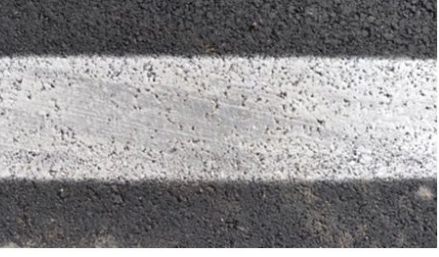

(b)
Fig. 9: (a) Dirt, (b) Tire marks

Structured dirt cannot be modeled because its structure is a priori unknown. So, this type of dirt is not considered in this study. Note that in specific configuration this dirt could be added manually by using dedicated software. Concerning the non-structured dirt, we can see in Fig. 10 that this dirt's spatial distribution is similar to that of the holes on the road. Accordingly, a similar model to the tear out model introduced in section IV.C is chosen. Indeed, spatial distribution is the same, so the same criteria to generate it can be used. A 2D Perlin noise $n(x, y)$ (scaled by an impact factor of $\operatorname{dirt}(\beta)$ is subtracted to the generated texture:

$$
p(x, y)=t(x, y)-\mathcal{J}_{d} n(x, y)
$$

3) Uniform wear

Uniform wear denotes all wear that uniformly impacts the entire surface of road marking. Two types of uniform wear can be enumerated: the more current one leads to road marking disappearance and the second one is due to paint penetration in the bitumen. The first type of wear occurs on top of gravel as shown in Fig. 10. The second one occurs between inter-gravel spaces as shown in Fig. 8 .

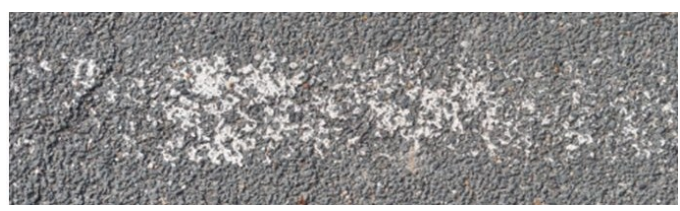

Fig. 10: Old road marking with time wear

The proposed uniform wear model is rather simple and efficient. It is based on the idea that the bitumen is monochrome. As a consequence, higher pixel values reflect more light than lowers ones. So, top of gravels is clear, and space between gravels is dark. Accordingly, a pixel below the low threshold $\left(L_{t h}\right)$ or above the high threshold $\left(H_{t h}\right)$ on texture gray level will be replaced by the corresponding pixel in the bitumen texture.

\section{E. Results and discussion}

The result of this process (implementation of filters) is a set of "shape-based" and "pixel-based" ground truth images with associated road marking textures. Each of them is labeled using four classes (label ' 0 ' for bitumen, '253' for left traffic lane, '254' for the middle one and ' 255 ' for the last one). This process generates several types of road marking depending of the parameters mentioned above. Note only suitable parameters allow generating a realistic road marking as shown in Fig 11a and Fig 11b.

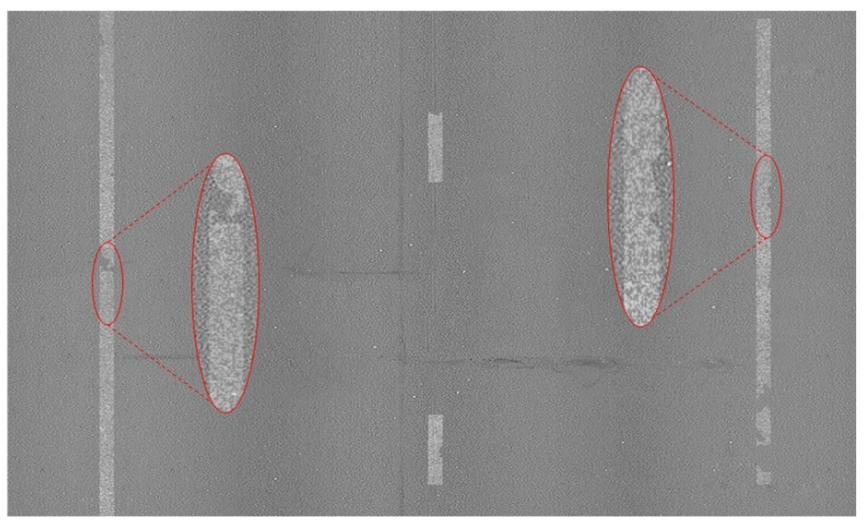

(a) 


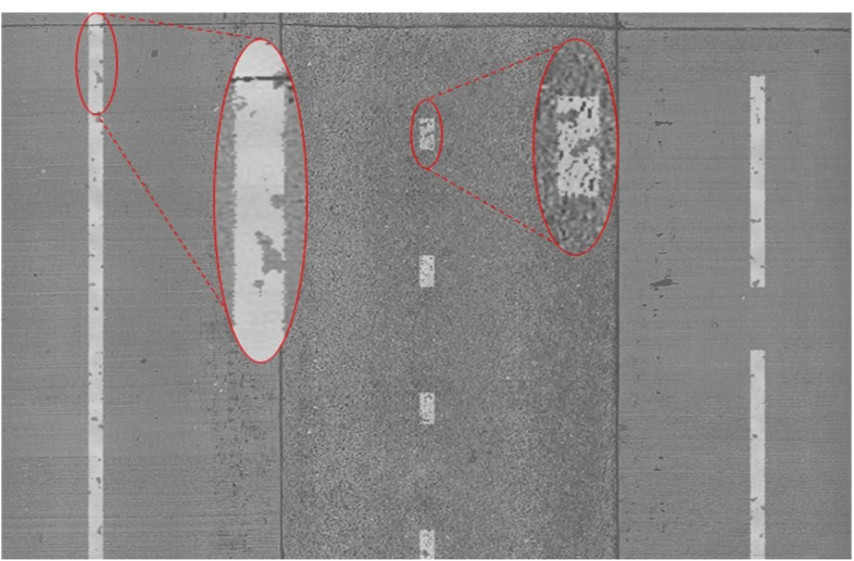

(b)

Fig 11: (a) and (b) Examples of road marking generation

The parameters used in order to generate the first road texture (presented in Fig 11a) are given in the first line of Tab 1. The second texture uses the parameters given in the second line.

\begin{tabular}{|c|c|c|c|c|c|c|c|c|c|c|c|c|}
\hline oct $_{h}$ & freq $_{h}$ per $_{h}$ & $t_{h}$ & $\mathcal{P}_{m}$ & $\mathcal{N}_{l}$ & $\mathcal{P}_{b}$ & oct $_{d}$ & freq $_{d}$ pers $_{d}$ & $\mathcal{J}_{d}$ & $L_{\text {th }}$ & $H_{\text {th }}$ \\
\hline 6 & 1 & 60 & -0.8 & 20 & 1 & 75 & 6 & 0.5 & 60 & 25 & 128 & 10 \\
\hline 6 & 6 & 50 & -0.7 & 70 & 1 & 65 & 6 & 2.5 & 40 & 16 & 190 & 100 \\
\hline
\end{tabular}

Tab 1: Parameters for texture generation. $\boldsymbol{o c t}_{\boldsymbol{h}}, \boldsymbol{f r e q}_{\boldsymbol{h}}, \boldsymbol{p} \boldsymbol{p r s}_{\boldsymbol{h}}, \boldsymbol{t}_{\boldsymbol{h}}$ : octave, frequency, persistence and threshold for holes. $\mathcal{P}_{m}, \mathcal{N}_{l}$ : proportion of modified pixels in the neighborhood,

$\mathcal{P}_{\boldsymbol{b}}$ : impact factor of the bitumen, $\boldsymbol{o c t}_{\boldsymbol{h}}, \boldsymbol{f r e q}_{\boldsymbol{h}}, \boldsymbol{p} \boldsymbol{e r} \boldsymbol{s}_{\boldsymbol{h}}, \boldsymbol{J}_{\boldsymbol{h}}$ : octave, frequency, persistence and impact for dirt, $\boldsymbol{L}_{\boldsymbol{t} \boldsymbol{h}}$ : wear due to a normal degradation $\boldsymbol{H}_{\boldsymbol{t} \boldsymbol{h}}$ : wear due to penetration of the paint.

From the texture shown in Fig 11a, some observation can be made: the texture has large holes due to low frequencies of the Perlin noise however their number is low $\left(\right.$ freq $_{h}=1$, $\left.t_{h}=-0.8\right)$; the edge are relatively straight $\left(\mathcal{P}_{m}=20\right.$ and $\mathcal{N}_{l}=$ $1)$; the stains are relatively strong and large $\left(\right.$ freq $_{d}=0.5$, $\left.\operatorname{pers}_{d}=60\right)$; the normal degradation is important, the road marking is fuzzy $\left(L_{t h}=190, H_{t h}=10\right)$.

In the texture shown in Fig $11 \mathrm{~b}$, the texture has small holes with high frequency, however their number is low $\left(\right.$ freq $\left._{h}=6, t_{h}=-0.7\right)$. The edge are blurred but the pixel dispersion is limited $\left(\mathcal{P}_{m}=70\right.$ and $\left.\mathcal{N}_{l}=1\right)$. The stains are relatively weak and small $\left(\right.$ freq $_{d}=2.5$, pers $\left._{d}=40\right)$. The paint penetration in the bitumen is weak but the road marking is clear $\left(L_{t h}=128, H_{t h}=100\right)$.

These examples adequately illustrate how much the Perlin noise is well-suited to generate realistic and varied marking textures.

\section{Performance EVAluation}

In some previous works [3], we proposed a performance evaluation protocol for the marking feature extraction step. In order to evaluate the impact of road marking wear on this extraction stage, performances are calculated with three scenarios (as illustrated in Fig 12):

a) road markings are new,

b) road markings are slightly worn,

c) road markings are highly worn.

d) real image of for comparison with our scenarios

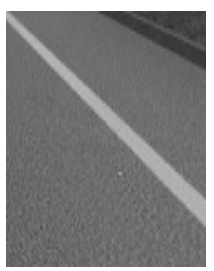

(a)

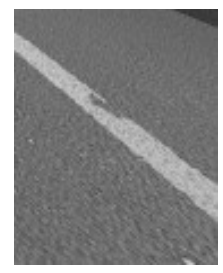

(b)

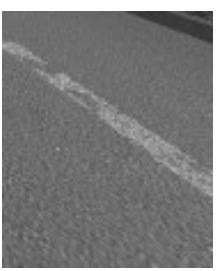

(c)

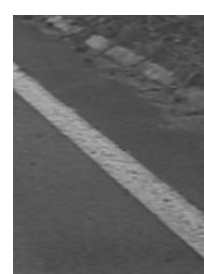

(d)
Fig 12: Illustration of the three scenarios of road marking wear and a real worn image for comparison (a) new (b)

slightly worn (c) highly worn (d) real worn image

Perlin parameters used to generate holes are the same for the three scenarios $\left(\right.$ oct $_{h}=6$, freq $_{h}=4$, pers $\left.s_{h}=20\right)$. Only the threshold $t_{h}$ is modified over scenarios as shown in Tab. 2 . With the value -1 for the new texture, all marking pixels are taken into account. The threshold values increases for slightly and highly worn textures meaning than less and less pixels are taken into account.

The smooth edges, the neighborhood size $\mathcal{N}_{l}$ is fixed to 1 for all scenarios, because applied this smooth filter on more than one pixel is visually unrealistic in our case. The proportion of modified pixels $\mathcal{P}_{m}$ increases with the wear rate. Perlin noise parameters for dirt effect are fixed to $\left(\right.$ oct $_{d}=6$, freq $_{d}=0.5$, pers $\left._{d}=60\right)$. The Impact of dirt $J_{d}$ increases with wear rate, although impact factor of bitumen $\mathcal{P}_{b}$ decreases. The interval of road marking paint penetration $\left[H_{t h}, L_{t h}\right]$ becomes narrower with wear rate.

\begin{tabular}{|c|c|c|c|c|c|c|}
\cline { 2 - 7 } \multicolumn{1}{c|}{} & $t_{h}$ & $\mathcal{P}_{m}$ & $\mathcal{P}_{b}$ & $\mathcal{J}_{d}$ & $L_{t h}$ & $H_{t h}$ \\
\hline (a) & -1 & 30 & 75 & 10 & 172 & 60 \\
\hline (b) & -0.75 & 50 & 70 & 20 & 160 & 70 \\
\hline (c) & -0.6 & 100 & 60 & 25 & 145 & 90 \\
\hline
\end{tabular}

Tab. 2: Parameters for texture generation applied to the three scenarios.

It is worth noting that all these parameters have been established by comparing the influence of parameters on synthesized images to real worn images.

Extraction results are shown with three extractors: MLT, SLT and PLT. More information on these extractors are given in [2].

Classically, as for any binary classification problem, performances are illustrated through the ROC and Dice curves. Their values are calculated through the study of the quadruplet $(T P, F P, T N, F N)$ representing respectively the True Positive, False Positive, True Negative and False Negative for different values of the threshold $T_{g}$.

ROC Curve is obtained by plotting the True Positive Rate (TPR) vs. the False Positive Rate (FPR) calculated as follows:

$$
\begin{aligned}
& T P R=\frac{T P}{T P+F N} \\
& F P R=\frac{F P}{F P+T N}
\end{aligned}
$$

As the main issue is to find a compromise between the TPR and the FPR, the DSC curve is complementary plotted as a function of $T_{g}$. Two criteria can help to understand DSC curve quality. Firstly, the peak value should be highest as possible, because it corresponds to an optimal value of the 
threshold $T_{g}$. Secondly, the width of the peak should be largest as possible, because it provides information on the extraction algorithm sensitivity to the threshold value.

In Fig 13 to ROC curves are plotted on top sub-figure and DICE curve on the bottom ones for $T_{g}=[1 ; 255]$ and with the minimum and maximum marking width $\left[S_{m}, S_{M}\right]=[10 ; 40]$ pixels.
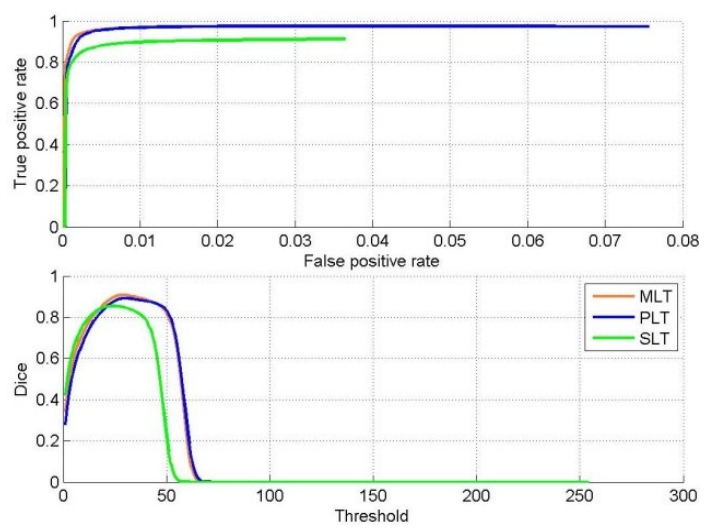

Fig 13: Extraction result with a new texture
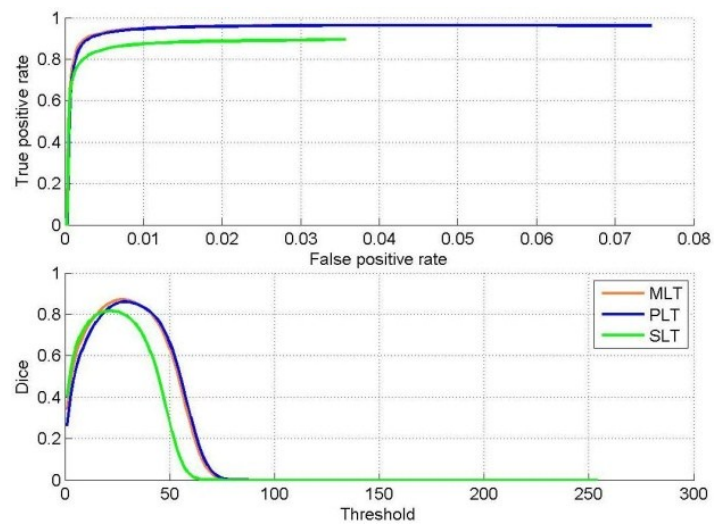

Fig 14: extraction result with a slightly worn texture
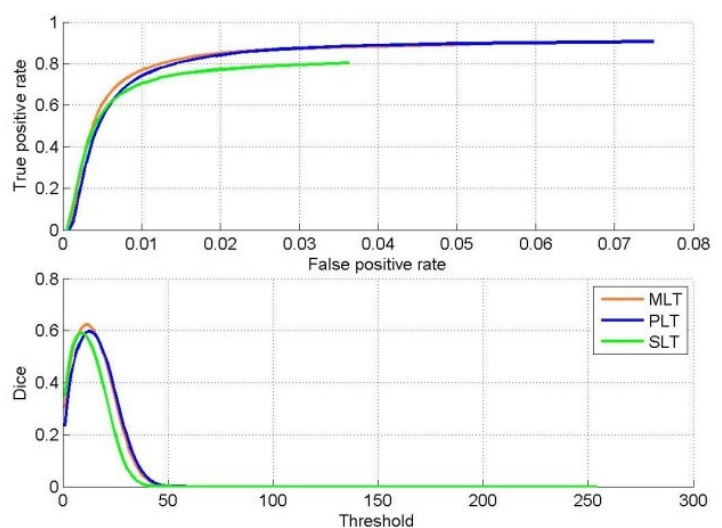

Fig 15: extraction result with a highly worn texture

When road marking are generated with a new texture, results are good; the maximum Dice value is more than 0.9 and is high for a wide range of $T_{g}$ value. In the slightly worn markings scenario, results are still good. The best maximum Dice value is 0.87 , and the peak is still quite large. However, with the highly worn texture, results are getting worse, the ROC curve increases very slowly and the maximum Dice value is only 0.62 . This result tends to show that our texture generator is efficient to create road marking with different levels of wear and different levels of imperfections. However, this result is not an absolute proof of the effectiveness of our generator. Indeed, it only shows the tendency of extractors to react to the quality of road markings. But, as the road markings wear is multi-factorial, defining reliable validation protocols is complicated.

\section{CONCLUSION AND FUTURE WORKS}

In this paper, we have proposed a generic tool for road marking generation dedicated to the traffic lanes. This tool is based on the use of natural bitumen textures. It takes into account both the type of traffic lanes and its wear. It also provides a shape-based and pixel-based ground truth that allows validating algorithms of road marking extraction.

This additional work for sensors simulation platform (SiVIC) can be considered enough reliable to evaluate and quantify the quality of road marking feature extraction algorithm. In its current state of development and with the use of Satory's test track scenario, this approach is both functional and operational. Indeed, the SiVIC simulator includes a realistic optical sensor, a geometric ground truth, realistic road marking textures and objects are correctly located on the tracks (buildings, trees, road signs, etc.).

This work can also be seen as the first step for dynamic generation of procedural textures for sensors and vehicle simulation platform. This approach and this texture generator will allow avoiding problems of repetitive textures for the traffic lanes.

\section{REFERENCES}

[1] S. Ieng, J. Vrignon, D. Gruyer, and D. Aubert, "A new multi-lanes detection using multi-camera for robust vehicle location," in Proc. of IEEE on Intelligent Vehicles Symposium. IV, 2005, pp. 700-705.

[2] T. Veit, J. Tarel, P. Nicolle, and P. Charbonnier, "Evaluation of road marking feature extraction", in Proc. of IEEE on International Conference on Intelligent Transportation Systems. ITSC, 2008, pp. 174-181.

[3] E. Pollard, D. Gruyer, J.P. Tarel, Sio-Song Ieng, A. Cord, "Lane marking extraction with combination strategy and comparative evaluation on synthetic and camera images" Proc. of IEEE on Conference on Intelligent Transportation Systems. ITSC, 2011, pp. 1741-1746.

[4] D. Gruyer, S. Glaser, B. Monnier, "SiVIC, a virtual platform for ADAS and PADAS prototyping, test and evaluation", accepted to FISITA'10, Budapest, Hungary, 30 may-4 june 2010.

[5] D. Gruyer, M. Grapinet, P. De Souza, "Modeling and validation of a new generic virtual optical sensor for ADAS prototyping", in IEEE Intelligent Vehicles Symposium, IV 2012, Alcalá de Henares, June 37, 2012, Spain,

[6] J. Sparbert, K. Dietmayer, and D. Streller, "Lane detection and street type classification using laser range images", in Proc of IEEE Intelligent Vehicles Symposium. IV, 2001, pp.454-459.

[7] J. McCall and M. Trivedi, "Video-based lane estimation and tracking for driver assistance: survey, system and evaluation," IEEE Transactions on Intelligent Transportation Systems, vol. 7, no. 1, pp. 20-37, 2006.

[8] E. Dumont, "Évaluation du degré d'usure des marquages routiers par traitement d'images", internal report of LCPC, unpublished. 2001

[9] K. Perlin, "An image synthesizer", in Proc of SIGGRAPH '85 Computer graphics and interactive techniques. SIGGRAPH, 1985, pp.287-296. 